\title{
Original
}

\section{High-Performance Liquid Chromatography with Multiple Coulometric Detector for Analysis of Active Substance Dynamics in Central Noradrenergic, Dopaminergic and Serotonergic Nervous Systems}

Akira Ohtsuki $^{1)}$, Hitomi Hasegawa ${ }^{1)}$, Hiroshi TaKeda ${ }^{2)}$ and Katsuji Oguchi ${ }^{1)}$

\begin{abstract}
A simple assay method for selective, highly sensitive, simultaneous determination of catecholamines, indoleamines and related metabolites in the central nervous system by high-performance liquid chromatography with a series of multiple coulometric detectors (HPLC-multiple coulometric detector) was investigated. For good separation of the analysanda (eleven compounds) and internal standards, a $\mu$ BONDAPAC $\mathrm{C}_{1 \text { s }}$ reversed-phase column and a mobile phase consisting of a $0.04 \mathrm{M}$ phosphate- $0.04 \mathrm{M}$ citrate mixed buffer ( $\mathrm{pH}$ 3.0) containing $7.5 \mathrm{mM}$ sodium 1-heptanesulphonate, $0.08 \mathrm{mM}$ ethylenediaminetetraacetic acid, $11.7 \%$ methanol and $4.7 \%$ acetonitrile were used. Also, the selective, highly sensitive and simultaneous detection of these neurochemical substances was performed with the redox-reductive screen mode using a series of three coulometric working electrodes (CWE). In this detection system, the first and second CWE were set at $+0.35 \mathrm{~V}$ and $+0.05 \mathrm{~V}$, respectively, for the prereaction and to prevent interference; the third CWE was used as an electrode for actual measurement, with its potential set at $-0.35 \mathrm{~V}$ against a palladium reference electrode. The assay limit was $0.5-5.0 \mathrm{pg}$. Excellent chromatograms of catecholamines, indoleamines and related metabolites were obtained within $25 \mathrm{~min}$. The usefulness of the HPLC-multiple coulometric detector system with the redox-reductive screen detection mode was confirmed by application to the determination of concentration levels of catecholamines, indoleamines and related metabolites in crude perchloric acid extracts of rat brain and spinal cord regions. These findings suggest that the HPLC-multiple coulometric detector system with the redox-reductive screen detection mode is useful for the study of neurochemical functions of active substances in the central noradrenergic, dopaminergic and serotonergic nervous systems.
\end{abstract}

Key words: HPLC, multiple coulometric detector, neurochemical substances, brain, spinal cord

\section{Introduction}

Neurochemical assessment of the dynamics of active substances in the central nervous system requires quantitative assay that is highly selective, sensitive, reproducible and reliable

1) Department of Pharmacology, Showa University School of Medicine, 1-5-8 Hatanodai, Shinagawa-ku, Tokyo 142, Japan.

2) Present Address; Department of Pharmacology, Tokyo Medical College, 6-1-1 Shinjuku, Shinjuku-ku, Tokyo 160, Japan. 
for the determination of these substances and their related metabolites and precursors in a single sample. High-performance liquid chromatography (HPLC) with electrochemical detection has become widely accepted for this purpose. At present, the combination of a thinlayer single amperometric detector with reversed-phase HPLC separation techniques is currently being used for analysis of neurochemical substances ${ }^{1-8)}$. This combination however, is not always satisfactory with respect to selectivity, sensitivity and reproducibility of the measurement of these substances by direct injection of crude biological samples into the HPLC apparatus ${ }^{9,10)}$. In particular, there are major shortcomings and problems with the detection limits in the high picogram to low nanogram range, and the inability to adequately detect the presence of coeluting substances.

Blank $^{11)}$ and Kissinger ${ }^{12)}$ devised the dual amperometric detection method for HPLC analysis. This detection method permits examination of different segments of the currentvoltage curve of each compound separated by HPLC. However, this technique has not completely solved the problems.

Recently, HPLC with a series of dual or multiple coulometric detectors (HPLC-dual or multiple coulometric detector) was applied to the measurement of neurochemically active substances in biological samples ${ }^{13-16)}$. It has also been reported that multiple coulometric detection methods with combination detection mode redox and reductive screen (redoxreductive screen detection mode) is suitable for selective, highly sensitive and reproducible detection of catechol, indole and o-methylated catechol compounds in crude perchloric acid extracts of biological samples ${ }^{1 i}$.

In the present study, a simple assay method for the selective, highly sensitive and simultaneous determination of catecholamines (norepinephrine, epinephrine, dopamine), indoleamine (serotonin) and related metabolites (4-hydroxy-3-methoxyphenylglycol, 3,4-dihydroxyphenylacetic acid, homovanillic acid, 3-methoxytyramine, 5-hydroxyindole-3-acetic acid) in rat brain and spinal cord regions by an HPLC-multiple coulometric detector system, with the redox-reductive screen detection mode, was investigated in detail.

\section{Materials and Methods}

\section{Animals}

Male Sprague-Dawley rats (Charles River Japan, Inc.) weighing 270-300 g were used. All animals were housed in groups of five per cage at constant room temperature $\left(23^{\circ} \mathrm{C}\right)$ and maintained on a $12 \mathrm{hr}$ light-dark cycle (the lighting period was from 7:00 to 19:00). Food and water were available ad libitum.

\section{Reagents}

All reagents were purchased at the highest available purity and used without further purification. Norepinephrine (NE), epinephrine (E), 3-hydroxytyramine hydrochloride (dopamine, DA), 5-hydroxytryptamine hydrochloride (serotonin, 5-HT), 4-hydroxy-3-methoxyphenylglycol (MHPG), 4-hydroxy-3-methoxyphenylacetic acid (homovanillic acid, HVA), 3methoxytyramine hydrochloride (3-MT), 5-hydroxyindole-3-acetic acid (5-HIAA) and deoxyepinephrine hydrochloride (DEP) were purchased from Sigma Chemical Co. (St. Louis). The reagents, 3,4-dihydroxyphenylacetic acid (DOPAC) and 5-hydroxy-Nw-methyltryptamine oxalate (n-MET) were obtained from Aldrich Chemical Co. (Milwaukee). Reagent grade chemicals for sample preparation and chromatography were obtained from Katayama Chemical Co. (Kyoto), and Nakarai Chemicals, Ltd. (Kyoto). 


\section{Sample preparation}

Rats were sacrificed by decapitation, and the brains and spinal cords were rapidly removed. The brains were dissected on a dry ice-cooled aluminum plate according to the method of Glowinski and Iversen ${ }^{18)}$, and the spinal cords were also dissected on the same plate. In this study, tissue samples of the striatum, hippocampus, hypothalamus and thoracic cord were used. After dissection, the tissue samples were immediately placed in liquid nitrogen and frozen until extraction.

Extraction was based on the procedure of Ikarashi and Maruyama ${ }^{i)}$. Dissected tissue was weighted and was initially added to a mixture of $1 \mathrm{ml}$ of $0.1 \mathrm{M}$ perchloric acid, $30 \mu \mathrm{l}$ of 0.1 $\mathrm{M}$ ethylenediaminetetraacetic acid, $30 \mu \mathrm{l}$ of $1 \mathrm{M}$ sodium hydrogen sulphite and $200 \mathrm{ng}$ each of DEP and n-MET as internal standards. The mixture was then homogenized with an ultrasonic cell disruptor (Sonifier model 200) at $13 \%$ power output $(20 \mathrm{~W})$ at $0^{\circ} \mathrm{C}$ for $30 \mathrm{sec}$ and centrifuged at $20000 \mathrm{~g}$ for $15 \mathrm{~min}$ at $0^{\circ} \mathrm{C}$. The supernatant layer was filtered through a $0.45 \mu \mathrm{m}$ Millipore filter to separate the insoluble residue. A portion of the supernatant (ca. $100 \mu \mathrm{l}$ ) was then carefully transferred to a small tube and further centrifuged at $20000 \mathrm{~g}$ for 2 min using a Beckman Microfuge. A portion of the supernatant was injected into the HPLC-multiple coulometric detector system.

HPLC-multiple coulometric detector system

\section{1) Apparatus}

The HPLC-multiple coulometric detector system is shown schematically in Fig. 1A. The HPLC system consisted of a Model LC-6A solvent delivery system (Shimazu Co., Kyoto) equipped with an extra dampener, a Model 7125 sample injector (Rheodyne Co., Cotati) with a $100 \mu \mathrm{l}$ sample holding loop, inline filter unit $(0.20 \mu \mathrm{m}$ graphite filter) (ESA) and a $\mu$ BONDAPAC $C_{18}$ reversed-phase column $(300 \mathrm{~mm} \times 3.9 \mathrm{~mm}$ I.D., $10 \mu \mathrm{m}$ particle size $)$ (Waters Assoc., Milford). A Model BX-7000 A column heater (Ishido Co., Chiba) was used to maintain the analytical column at a constant temperature.

Electrochemical detection was carried out with a coulometric detection system consisting of three coulometric high-efficiency flow-through cells in series. This detection system consisted of a Model 5021 conditioning cell with a single coulometric working electrode [test electrode 1; $\mathrm{T}_{1}$ (efficiency 100\%)] $(\mathrm{ESA})^{14)}$, a Model 5011 high-sensitivity analytical cell containing dual coulometric working electrodes [test electrode $2 ; \mathrm{T}_{2}$ (efficiency $100 \%$ ), test electrode 3; $\mathrm{T}_{3}$ (efficiency $\left.70 \%\right)$ ] (ESA) ${ }^{14-16)}$ and a Model 5100A control module (ESA). The three coulometric working electrodes $\left(T_{1}, T_{2}\right.$ and $\left.T_{3}\right)$ were connected in series by installing a Model 5021 conditioning cell before the Model 5011 high-sensitivity analytical cell. Data analysis was performed with a Model 12 SIC chromatocorder (SIC) connected to $\mathrm{T}_{3}$.

2) Electrochemical detection mode

The principle of the electrochemical detection mode of this HPLC-multiple coulometric detector system is illustrated in Fig. 1B. For the selective, highly sensitive and simultaneous detection of catecholamines, indoleamines and related metabolites, the redox-reductive screen detection mode ${ }^{1 i}$ ) was performed using a series of three coulometric working electrodes. In this detection mode, the first high-efficiency electrode $\left(T_{1}\right)$ was set at $+0.35 \mathrm{~V}$ corresponding to the top of the oxidation current-voltage curve of the analysandum, to completely oxidize these compounds. The second $\left(\mathrm{T}_{2}\right)$ and third electrodes $\left(\mathrm{T}_{3}\right)$ were set at different reduction potentials. The potential of $\mathrm{T}_{2}$ was set at $+0.05 \mathrm{~V}$, near the low end of the reduction 
current-voltage curve of the analysandum, to remove compounds having lower reduction potentials than the analysanda. Also, the potential of the third electrode $\left(T_{3}\right)$ was set at $-0.35 \mathrm{~V}$, corresponding to the top of the curve for the actual measurement of the analysandum. 3) Mobile Phase

For good separation of catecholamines, indoleamines, related metabolites and internal standards, a mobile phase consisting of a $0.04 \mathrm{M}$ phosphate- $0.04 \mathrm{M}$ citrate mixed buffer containing $0.08 \mathrm{mM}$ ethylenediaminetetraacetic acid, ion-pair agent and organic solvents was used. The mobile phase flow-rate was maintained at $1.0 \mathrm{ml} / \mathrm{min}$. Mobile phase was also circulated in the HPLC-multiple coulometric detector system and was exchanged when marked changes in the back-current occurred.

(A)

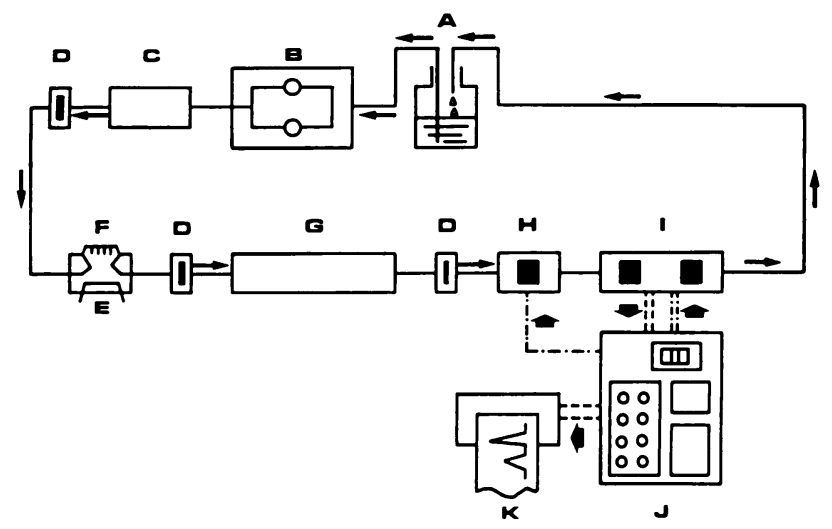

(B)
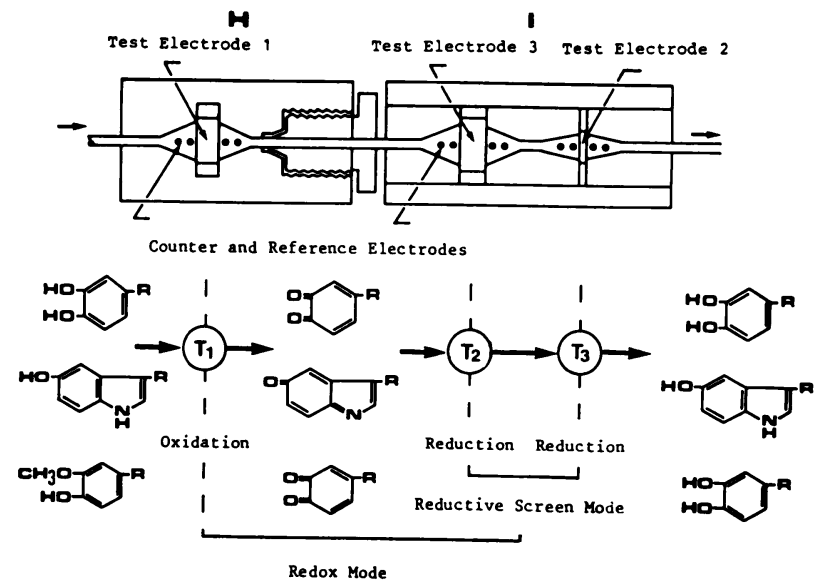

Fig. 1. HPLC-multiple coulometric detector system and detection mode for the determination of catecholamines, indoleamines and related metabolites.

(A) HPLC-multiple coulometric detector system and (B) detection mode.

A: mobile phase, B: pump, C: damper, D: in-line filter, E: injection valve, F: sampling loop, G: analytical column, H: Model 5021 conditioning cell (ESA), I: Model 5011 high sensitivity analytical cell (ESA), J: Model 5100A control module (ESA), $\mathrm{K}$ : Model 12 SIC chromatocorder (SIC).

T1, T2 and T3: test electrodes 1,2 and 3, respectively. 


\section{Results}

\section{Optimum condition of separation}

To determine the optimum condition for good separation of catecholamines, indoleamines, related metabolites and internal standards, the effects of four mobile phase parameters and the maintenance temperature of the analytical column on capacity factor $\left(\mathrm{K}^{\prime}\right)$ values for individual compounds were investigated.

(A) SHS

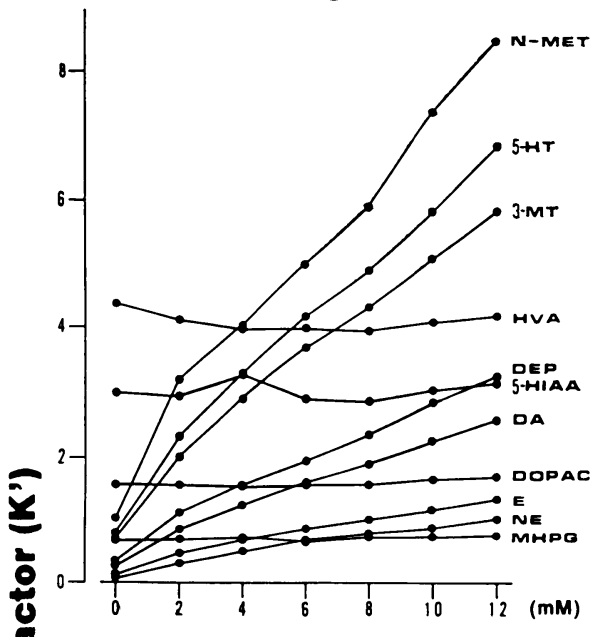

(B) PH

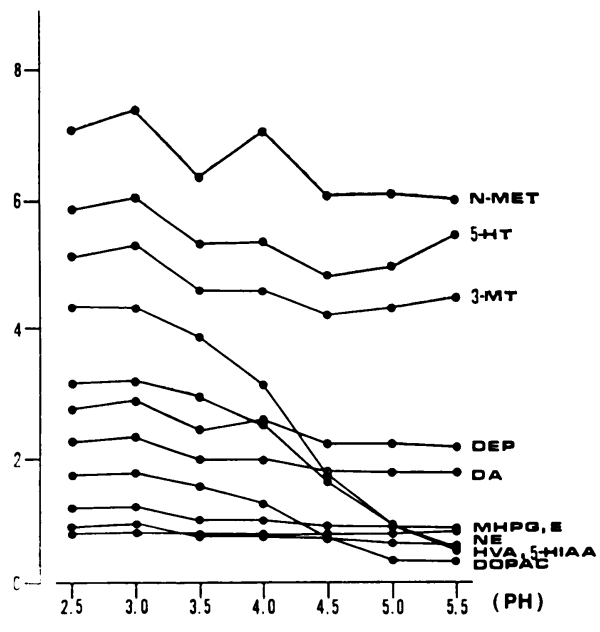

(C)Methanol

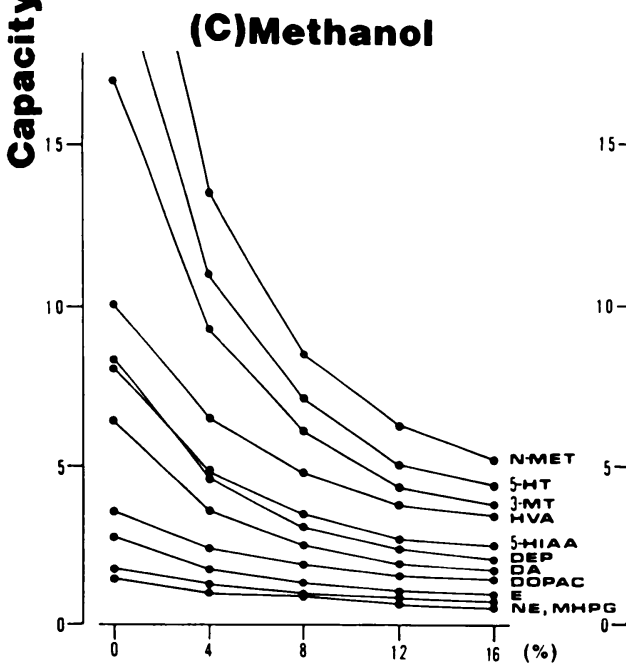

(D) Acetonitrile

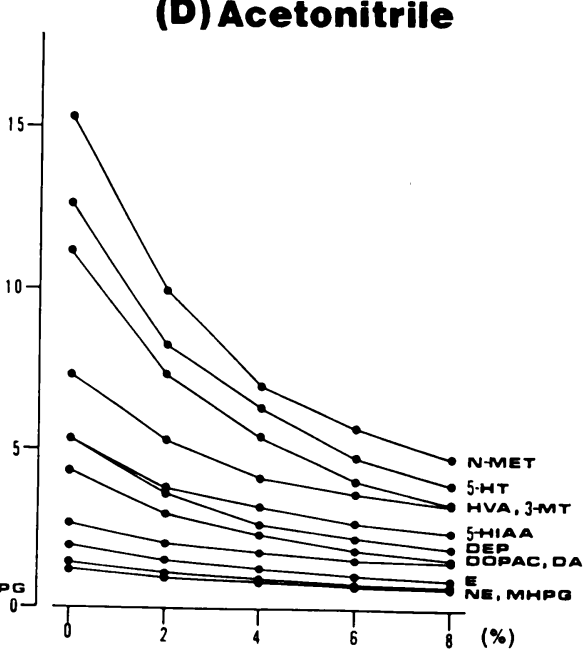

Fig. 2. Effects of mobile phase parameters on capacity factor for catecholamines, indoleamines and related metabolites.

(A) Sodium 1-heptanesulphonate (SHS), (B) pH, (C) methanol and (D) acetonitrile. MHPG: 4-hydroxy-3-methoxyphenylglycol, NE: norepinephrine, E: epinephrine, DOPAC: 3,4-dihydroxyphenylacetic acid, DA: dopamine, DEP: deoxyepinephrine, 5-HIAA: 5-hydroxyindole-3-acetic acid, HVA: homovanillic acid, 3-MT: 3-methoxytyramine, 5-HT: serotonin, n-MET: 5-hydroxy-Nw-methyltryptamine. 
The effect of sodium 1-heptanesulphonate on $\mathrm{K}^{\prime}$ values is shown in Fig. 2A. The $\mathrm{K}^{\prime}$ values for basic compounds (n-MET, 5-HT, 3-MT, DEP, DA, E and NE) were markedly enhanced with increasing concentrations of sodium 1-heptanesulphonate, while neutral (MHPG) and acidic compounds (HVA, 5-HIAA and DOPAC) were not affected in the concentration range of 0 to $12 \mathrm{mM}$. The effect of $\mathrm{pH}$ in the mobile phase on $\mathrm{K}^{\prime}$ values is shown in Fig. 2B. Changing $\mathrm{pH}$ from 3.5 to 5.5 produced dramatic decreases in the $\mathrm{K}^{\prime}$ values for acidic compounds. On the other hand, the $\mathrm{K}^{\prime}$ values for basic and neutral compounds were unaffected in the $\mathrm{pH}$ range of 2.5 to 5.5. For all compounds examined, the $\mathrm{K}^{\prime}$ values were gradually decreased by raising concentrations of methanol or acetonitrile in the mobile phase Figs. 2C, D. The effect of the temperature in the analytical column on $\mathrm{K}^{\prime}$ values is shown in Fig. 3. In the temperature range of 23 to $45^{\circ} \mathrm{C}$, the $\mathrm{K}^{\prime}$ values for basic, neutral and acidic compounds decreased with an increase in temperature.

From these results, the mobile phase composition for good separation of catecholamines, indoleamines, related metabolites and internal standards was the $0.04 \mathrm{M}$ phosphate-0.04 $\mathrm{M}$ citrate mixed buffer, $\mathrm{pH} 3.0$, containing $7.5 \mathrm{mM}$ sodium 1-heptanesulphonate, $0.08 \mathrm{mM}$ ethylenediaminetetraacetic acid, $11.7 \%$ methanol and $4.7 \%$ acetonitrile. Also, the most suitable temperature of the analytical column was $23^{\circ} \mathrm{C}$ with this mobile phase composition. Accuracy of measurement

The calibration curves for determination of catecholamines, indoleamines and related metabolites in brain or spinal cord regions are shown in Fig. 4. These curves were obtained by plotting the peak height ratios of individual analytes to internal standards versus the amounts of analysanda. There was close correlation $(r=0.9994 \sim 0.9999)$ between the peak

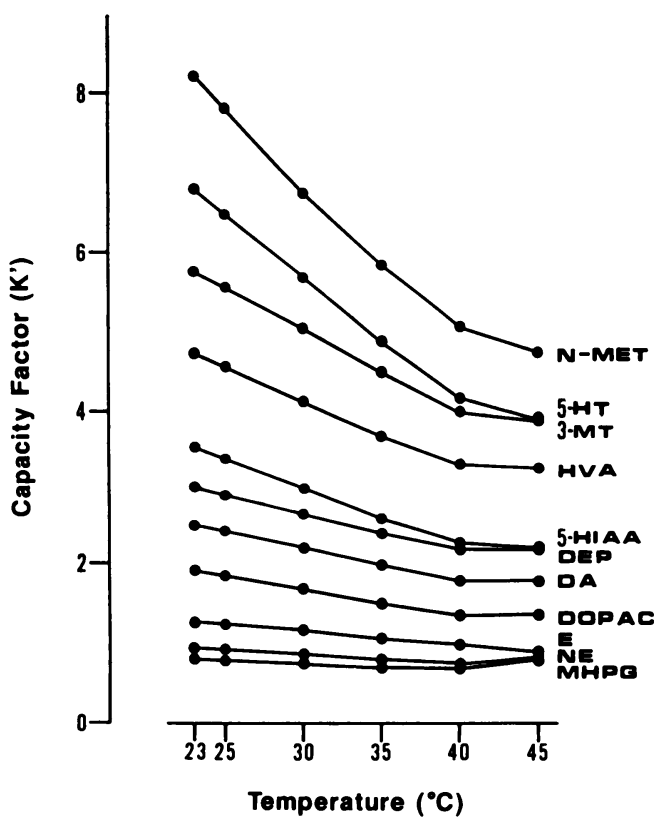

Fig. 3. Effect of temperature of analytical column on capacity factor for catecholamines, indoleamines and related metabolites.

Other explanations as for Fig. 2. 


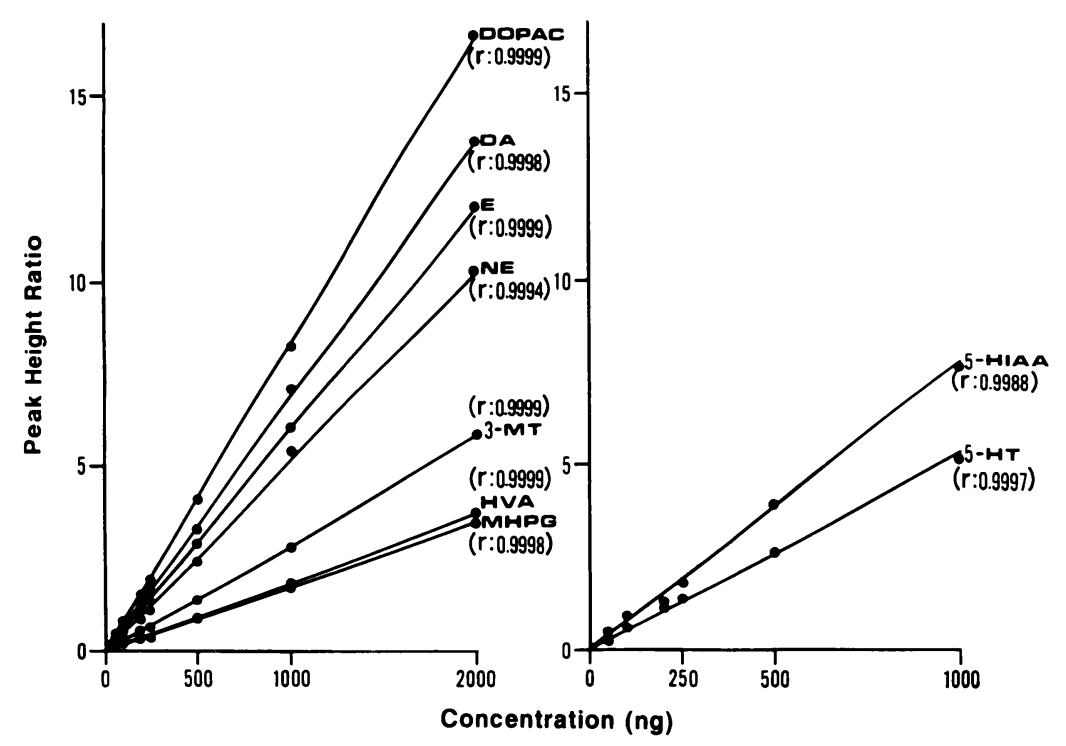

Fig. 4. Calibration curves for the determination of catecholamines, indoleamines and related metabolites. Peak height ratios are with respect to a fixed amount of internal standard (DEP for NE, E, DA, MHPG, DOPAC, HVA and 3-MT, n-MET for 5-HT and 5-HIAA).

Other explanations as for Fig. 2.

height ratios of NE, E, DA, DOPAC, MHPG, 3-MT or HVA to DEP and the amounts of these compounds. It was also confirmed that the peak height ratios of 5-HT or 5-HIAA to $n-M E T$ and the amounts of these compounds were linearly correlated $(r=0.9988 \sim 0.9997)$.

The assay limits for catecholamines, indoleamines and related metabolites by HPLCmultiple coulometric detector system were rather compound dependent. The assay limit for each analysandum (with a signal-to-noise ratio of 2) was as follows: 2.1 (MHPG), 0.6 (NE), 0.6 (E), 0.5 (DOPAC), 0.5 (DA), 2.4 (5-HIAA), 5.0 (HVA), 1.7 (3-MT) and 3.2 pg (5-HT).

\section{Chromatograms}

Representative chromatograms obtained by direct injection of standard solution or perchloric acid extract of rat striatum tissue into the HPLC-multiple coulometric detector system are shown in Fig. 5. Excellent chromatograms of catecholamines, indoleamines and related metabolites were obtained within $25 \mathrm{~min}$, and the large 'void volume' signal and baseline drift typically observed in the chromatogram after direct injection of perchloric acid extracts were markedly reduced.

Regional levels of catecholamines, indoleamines and related metabolites in rat brain and spinal cord

The concentrations of catecholamines, indoleamines and related metabolites in striatum, hypothalamus, hippocampus and thoracic cord are shown in Table 1. The levels of DA, DOPAC, 3-MT and HVA concentrations in striatum were highest in the regions examined. In the hypothalamus, the levels of NE, E, 5-HT and 5-HIAA concentrations were higher than in other regions. Also, 3-MT concentration in the hippocampus and 3-MT and HVA concentrations in the thoracic cord could not be determined, due to lack of sensitivity. 


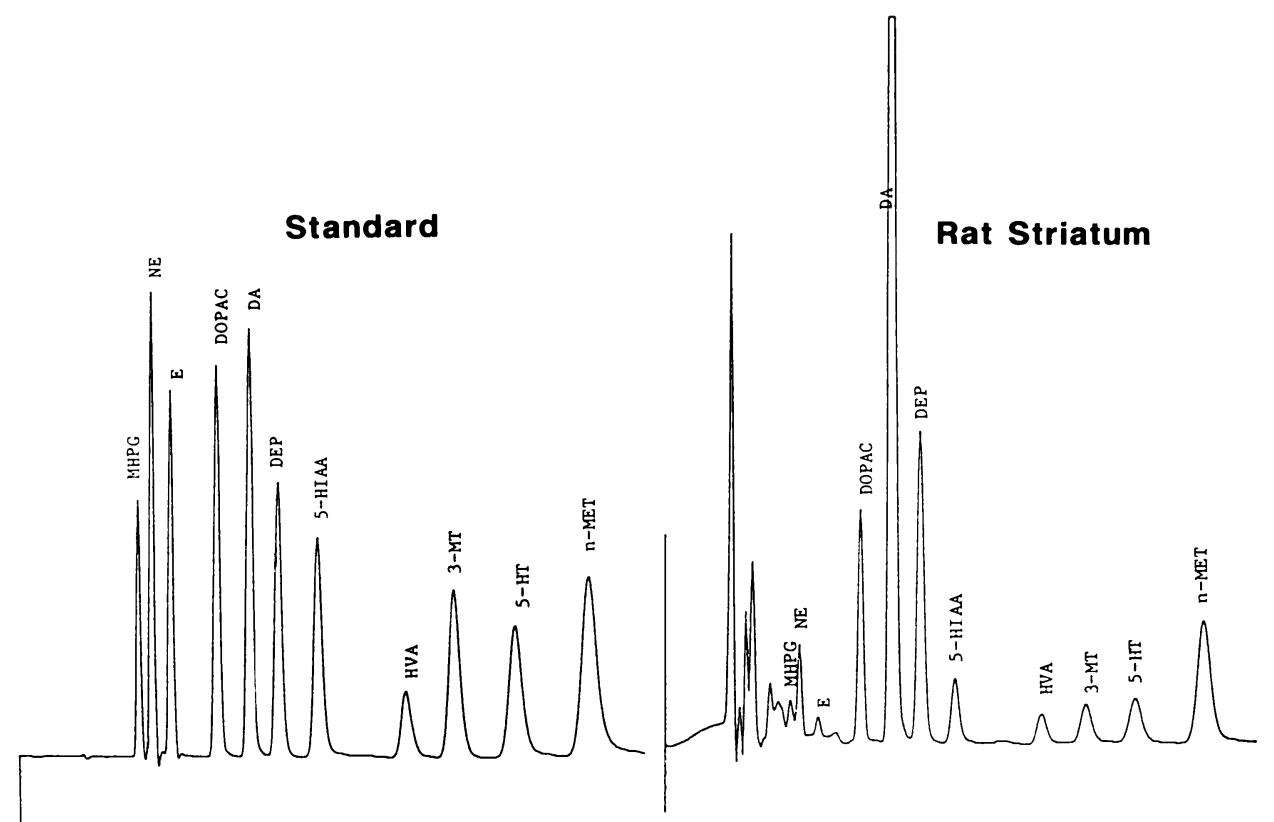

Fig. 5. Representative chromatogram obtained by direct injection of standard solution or perchloric acid extract of rat striatum tissue into HPLC-multiple coulometric detector system with the redox-reductive screen detection mode. Chromatographic conditions: analytical column; $\mu$ BONDAPAC $C_{1 \kappa}(300 \mathrm{~mm} \times 3.9 \mathrm{~mm}$ I.D. $)$, maintenance temperature of analytical column; $23^{\circ} \mathrm{C}$, flow-rate; $1.0 \mathrm{ml} / \mathrm{min}$, mobile phase; mixed $0.04 \mathrm{M}$ phosphate- $0.04 \mathrm{M}$ citrate buffer, $\mathrm{pH} 3.0$, containing $7.5 \mathrm{mM}$ sodium 1heptanesulphonate, $0.08 \mathrm{mM}$ ethylenediaminetetraacetic acid, $11.7 \%$ methanol and $4.7 \%$ acetonitrile. Applied potential: test electrode $1 ;+0.35 \mathrm{~V}$, test electrode 2; $+0.05 \mathrm{~V}$ and test electrode $3 ;-0.35 \mathrm{~V}$.

Other explanations as for Fig. 2.

Table 1. Concentrations of catecholamines, indoleamines and related metabolites in rat brain and spinal cord regions.

\begin{tabular}{lcccc}
\hline & Striatum & Hypothalamus & Hippocampus & Thoracic cord \\
\hline NE & $340 \pm 48$ & $1531 \pm 203$ & $383 \pm 45$ & $265 \pm 32$ \\
MHPG & $92 \pm 10$ & $82 \pm 23$ & $53 \pm 12$ & $25 \pm 16$ \\
E & $5 \pm 2$ & $34 \pm 5$ & $15 \pm 5$ & $3 \pm 1$ \\
DA & $7250 \pm 196$ & $553 \pm 61$ & $75 \pm 10$ & $55 \pm 6$ \\
DOPAC & $976 \pm 81$ & $102 \pm 14$ & $36 \pm 9$ & $7 \pm 3$ \\
3-MT & $283 \pm 80$ & $9 \pm 2$ & N.D. & N.D. \\
HVA & $810 \pm 96$ & $6 \pm 1$ & $15 \pm 8$ & N.D. \\
5-HT & $814 \pm 98$ & $1102 \pm 78$ & $681 \pm 73$ & $407 \pm 60$ \\
5-HIAA & $683 \pm 83$ & $751 \pm 82$ & $537 \pm 40$ & $215 \pm 59$
\end{tabular}

Unit: ng/g, N.D.: Not detectable. Each value represents the mean \pm S.D. for 6 animals. Other explanations as for Fig. 2. 


\section{Discussion}

For proper evaluation of the neurochemical functions of active substances in the central noradrenergic, dopaminergic and serotonergic nervous systems, it is necessary to establish a quantitative assay method with reproducibility and reliability for the selective, highly sensitive simultaneous determination of NE, DA, 5-HT and major metabolites and precursors of their neurotransmitters.

The dual or multiple coulometric detection method for quantitative HPLC was recently introduced and applied to measuring catecholamines, indoleamines and their metabolites and precursors in crude biological samples, such as unprocessed cerebrospinal fluid and perchloric acid extracts of brain tissues ${ }^{13-16)}$. This system greatly enhanced the sensitivity, selectivity and reproducibility of these analyses. In 1990, Takeda et al. ${ }^{17}$ ) investigated a suitable detection mode for the determination of catecholamines, indoleamines and related metabolites in crude perchloric acid extracts of biological samples by the HPLC-multiple coulometric detector system, and reported that for the highly sensitive detection of catechol compounds, the oxidative single, redox and redox-reductive screen modes are suitable. Also, the oxidative single and oxidative screen modes are better than the redox or redox-reductive screen modes for indole and o-methylated catechol compounds. For selective and simultaneous detection of these compounds in crude perchloric acid extracts of biological samples; however, the redox-reductive screen mode was the best of the four detection modes examined.

In this study, we investigated a simple assay method for the selective, highly sensitive, simultaneous determination of catecholamines, indoleamines and related metabolites by direct injection of crude perchloric acid extracts of brain or spinal cord regions into the HPLCmultiple coulometric detector system with the redox-reductive screen detection mode.

It is known that the effects of organic solvent, ion-pair agent, and $\mathrm{pH}$ of the mobile phase on capacity factor $\left(\mathrm{K}^{\prime}\right)$ of solutes are primarily caused by modifying the solubility of hydrophobic solute molecules, protonation of the solute, and formation of ion-pairs between the solute and the agent in the mobile phase ${ }^{7,19-21)}$. In the present study, to find the optimum condition for good separation of catecholamines, indoleamines, related metabolites and internal standards, the effects of four mobile phase parameters (concentration of sodium 1-heptanesulphonate, methanol or acetonitrile, and $\mathrm{pH}$ ) and the maintenance temperature of the analytical column on $\mathbf{K}^{\prime}$ values for individual compounds were investigated. The results suggest that the combination of a $\mu$ BONDAPAC $C_{18}$ reversed-phase column and a mobile

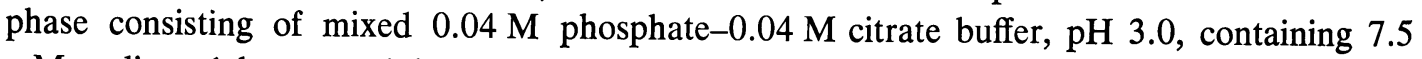
$\mathrm{mM}$ sodium 1-heptanesulphonate, $0.08 \mathrm{mM}$ ethylenediaminetetraacetic acid, $11.7 \%$ methanol and $4.7 \%$ acetonitrile is suitable. It is also necessary to maintain the analytical column at the constant temperature of $23^{\circ} \mathrm{C}$.

In this study, the redox-reductive screen mode, employing a series of three coulometric working electrodes, was used as the detection mode of the HPLC-multiple coulometric detector system. In this detection mode, the first high-efficiency coulometric working electrode was set at $+0.35 \mathrm{~V}$ to oxidize most of the analysandam eluted from the column. This improves the selectivity by eliminating the detection of non-reversible substances at subsequent electrodes operated in the reductive mode. Thus, substances that can be completely oxidized at $+0.35 \mathrm{~V}$ and cannot be subsequently reduced appear in the analysis with the recording electrode set at $-0.35 \mathrm{~V}$. Secondly, oxidation of the column effluent greatly reduces base- 
line drift and virtually eliminates the large void currents routinely observed with amperometric detectors which often direct injection of crude perchloric acid extracts into the HPLC apparatus. The second coulometric working electrode was set at $+0.05 \mathrm{~V}$ to eliminate the detection at subsequent recording electrodes of reversible substances having a lower reduction potential than the analysandum. The third electrode was set at $-0.35 \mathrm{~V}$ for the actual measurement of the analyte.

The results of the present study suggest that an HPLC-multiple coulometric detector system, with a redox-reductive screen detection mode, assembled on the basis of the experimental results has several advantages: First, highly sensitive and simultaneous determination of catecholamines, indoleamines and related metabolites is possible due to assay limits in the high femtogram to low picogram range. Secondly, the quantitative analysis of early eluting substances such as MHPG and NE is possible due to significant reduction in the 'void volume' signal, the virtual elimination of interference from non-reversible substances, and the stabilization of the baseline signal. Thirdly, the measurement of catecholamines, indoleamines and related metabolites by direct injection of crude perchloric acid extracts is possible. This greatly improves the analysis efficiency, because it eliminates errors in sample preparation procedures and corrections based on recovery rates. The usefulness of the HPLCmultiple coulometric detector system with the redox-reductive screen detection mode was confirmed by its application in the simultaneous determination of various neurochemicals in rat striatum, hypothalamus, hippocampus and thoracic cord.

In conclusion, the HPLC-multiple coulometric detector system with the redox-reductive screen detection mode is useful in the study of the dynamics of neurochemically active substances, including turnover in the central noradrenergic, dopaminergic and serotonergic nervous systems and interactions between these systems.

\section{References}

1) Hefti F: A simple, sensitive method for measuring 3,4-dihydroxyphenylacetic acid and homovanillic acid in rat brain tissue using high-performance liquid chromatography with electrochemical detection. Life Sci, 25: 775-782 (1979)

2) Magnusson $O$, Nilsson LB and Westerlund D: Simultaneous determination of dopamine, DOPAC and homovanillic acid. Direct injection of supernatant from brain tissue homogenates in liquid chromatography electrochemical detection system. J Chromatogr, 221: 237-247 (1980)

3) Mefford IN and Barchas JD: Determination of tryptophan and metabolites in rat brain and pineal tissue by reversed-phase high-performance liquid chromatography with electrochemical detection. $J$ Chromatogr, 181: 187-193 (1980)

4) Wagner J, Vitali P, Palhreyman MG, Zraika $M$ and Huot S: Simultaneous determination of 3,4dihydroxyphenylalanine, 5-hydroxytryptophan, dopamine, 4-hydroxy-3-methoxyphenylalanine, norepinephrine, 3,4-dihydroxyphenylacetic acid, homovanillic acid, serotonin, and 5-hydroxyindoleacetic acid in rat cerebrospinal fluid and brain by high-performance liquid chromatography with electrochemical detection. J Neurochem, 38: 1241-1254 (1982)

5) Causon RC and Carruthers ME: Measurement of catecholamines in biological fluids by highperformance liquid chromatography. A comparison of fluorimetric with electrochemical detection. J Chromatogr, 229: 301-309 (1982)

6) Lin PYT, Bulawa MC, Wong P, Lin L, Scott J and Blank CL: The determination of catecholamines, indoleamines, metabolites, and related enzymatic activities using three micron liquid chromatography columns. J Liq Chromatogr, 7: 509-538 (1984)

7) Ikarashi $Y$ and Maruyama $Y$ : High-performance liquid chromatographic analysis of regional catecholamines and 3,4-dihydroxyphenylacetic acid in rat brain following microwave irradiation. Biogenic Amines, 1: 341-357 (1984) 
8) Ikarashi $Y$ and Maruyama $Y$ : Determination of catecholamines, indoleamines, and related metabolites in rat brain with liquid chromatography with electrochemical detection. Biogenic Amines, 2: 101110 (1985)

9) Langlais PJ, McEntee WJ and Bird ED: Rapid liquid chromatographic measurement of 3-methoxy4-hydroxyphenylglycol and other monoamine metabolites in human cerebrospinal fluid. Clin Chem, 26: $786-788$ (1980)

10) Langlais PJ, Bird ED and McEntee WJ: Stability of monoamine metabolites in human cerebrospinal fluid. Ann Neurol, 12: 48-51 (1982)

11) Blank CL: Dual electrochemical detector for liquid chromatography. J Chromatogr, 117: 35-46 (1976)

12) Kissinger PT: Amperometric and coulometric detectors for high performance liquid chromatography. Anal Chem, 49: 447-456 (1977)

13) Dutrieu J and Delmotte YA: Dual electrochemical detection in HPLC, new trend in analytical chemistry. Fresenius $Z$ Anal Chem, 314: 416-417 (1983)

14) Langlais PJ, Bird ED and Matson WR: An automated HPLC, three cell electrochemical method for the simultaneous assay of monoamines and metabolites in crude brain extracts. Clin Chem, 30: 1046 (1984a)

15) Matson WR, Langlais PJ, Volicer L, Gamache PH, Bird ED and Mark KA: n-Electrode three dimensional liquid chromatography with electrochemical detection for determination of neurotransmitters. Clin Chem, 30: 1477-1488 (1984)

16) Kilpatrick IC, Jones MW and Phillipson OT: A semiautomated analysis method for catecholamines, indoleamines, and some prominent metabolites in microdissected regions of the nervous system: an isocratic HPLC technique employing coulometric detection and minimal sample preparation. J Neurochem, 46: 1865-1876 (1986)

17) Takeda $H$, Matsumiya $T$ and Shibuya $T$ : Detection and identification modes for the highly sensitive and simultaneous determination of various biogenic amines by coulometric high-performance liquid chromatography. J Chromatogr, 515: 265-278 (1990)

18) Glowinski $J$ and Iversen LL: Regional studies of catecholamines in the rat brain-1: The disposition of ${ }^{3} \mathrm{H}$-norepinephrine, ${ }^{3} \mathrm{H}$-dopamine and ${ }^{3} \mathrm{H}$-dopa in various regions of the brain. $\mathrm{J}$ Neurochem, 13: 655-669 (1966)

19) Bidlingmeyer BA: Retention mechanism for reversed-phase ion-pair liquid chromatography. J Chromatogr, 186: 419-434 (1979)

20) Bidlingmeyer BA: Separation of ionic compounds by reversed-phase liquid chromatography. An update of ion-pairing techniques. J Chromatogr Sci, 18: 525-539 (1980)

21) Krstulović AM: Investigations of catecholamine metabolism using performance liquid chromatography. J Chromatogr, 229: 1-34 (1982a)

[Received February 1, 1991: Accepted February 25, 1991] 